\title{
[4+2] CYCLOADDITION OF N-ETHOXYCARBONYLAZEPINE AND
}

5, 5-DIMETHOXY-1, 2, 3, 4-TETRACHIOROCYCLOPENTADIENE

John R. Wlseman and Berni P. Chong

Dept. of Chemistry, Univ. of Michigan, Ann Arbor, Michigan 48104

(Received in USA 15 February 1969; received in UK for publication 25 March 1969)

Cycloadition reactions of azepines have recently generated considerable interest. Three types of cycloaddition reactions involving azepines have been reported: a $[4+2]$ cycloaddition of N-ethoxycarbonylazepine with tetracyanoethylene $e^{1}$ in which the azepine behaves as a diene, [6+4] dimerizations ${ }^{2-4}$ in which the azepines serve as both diene and triene, and a reaction formulated as a [6+2] cycloaddition of N-ethoxycarbonylazepine with nitrosobenzene. 5 We wish to report the observation of a new type of cycloaddition, a [4+2] reaction in which the 4,5 double bond of N-ethoxycarbonylazepine behaves as a dienophile. 6 When N-ethoxycarbonylazepine (I) and 5,5-dimethoxy-1,2,3,4-tetrachlorocyclopentadiene (II) were heated to $100^{\circ} \mathrm{C}$ for four hours, a colorless, vis-

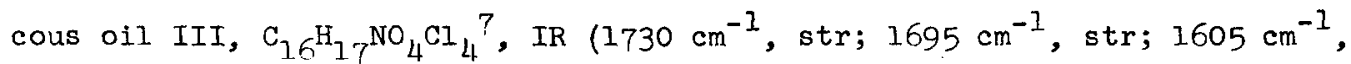
med), was isolated by column chromatography in $30 \%$ yield. The simple NMR spectrum, $\tau 3.16(2 \mathrm{H}, \mathrm{d}, \mathrm{J}=11 \mathrm{~Hz}), \tau 5.15$ (2H, broad d, J=11Hz), $\tau 5.82(2 \mathrm{H}, \mathrm{q}$, $\mathrm{J}=7 \mathrm{~Hz}), \tau 6.45(3 \mathrm{H}, \mathrm{s}), \tau 6.52(3 \mathrm{H}, \mathrm{s}), \tau 6.67$ (2H, broad s), $\tau 8.68$ (3H, $t$, $J=7 \mathrm{~Hz})$, demands a symmetrical structure and is consistent with both IIIa and IIIb. The UV spectrum of III $\left(\lambda_{\max }=226 \mathrm{m \mu}, \varepsilon=1.2 \times 10^{4}\right)$ is nearly 1dentical to that of VI $\left(\lambda_{\max }=228 \mathrm{m \mu}, \varepsilon=1.23 \times 10^{4}\right)^{8}$ which contains the same chromophore as IIIb. In contrast, the UV spectrum of VII ( $\lambda_{\max }=257$ and 267 $\mathrm{m \mu}, \varepsilon=7680$ and 7870$)^{9}$ which contains a chromophore similar to IIIa is very different from the UV spectrum of III.

Hydrogenation of III at $50 \mathrm{lbs}$. pressure for 24 hours with a $5 \% \mathrm{Pd} / \mathrm{C}$ catalyst yielded a crystalline tetrahydro compound IV, $\mathrm{C}_{16} \mathrm{H}_{21} \mathrm{NO}_{4} \mathrm{Cl}_{4}$, $\mathrm{mp} 106.5-$ $108.5^{\circ} \mathrm{C}$, IR $\left(1605 \mathrm{~cm}^{-1}, \mathrm{str}\right), \operatorname{MR} \tau 5.93(4 \mathrm{H}, \mathrm{q}, \mathrm{J}=7 \mathrm{~Hz}$, superimposed on a multiplet), $\tau 6.44$ ( $3 \mathrm{H}, \mathrm{s}), \tau 6.50$ (3H, s), $\tau 7.21$ (4H, multiplet), $\tau 8.00$ 


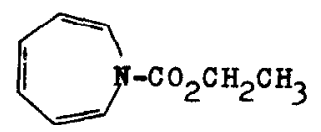

I

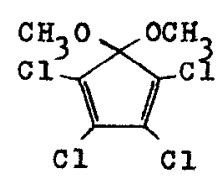

II

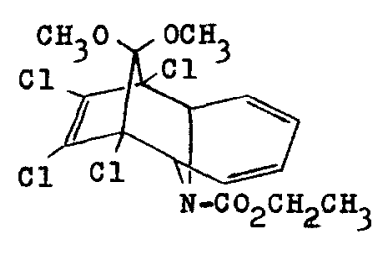

III

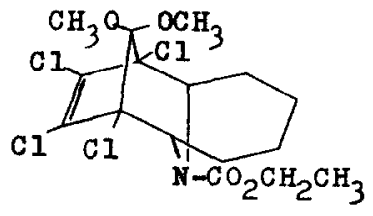

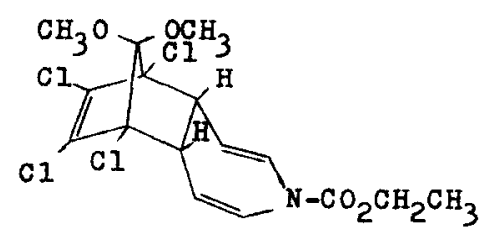

IIIb

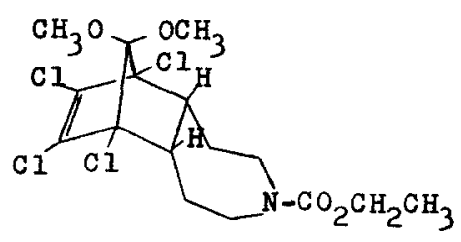

IVb

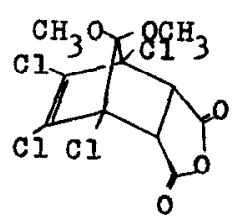

v
IVa

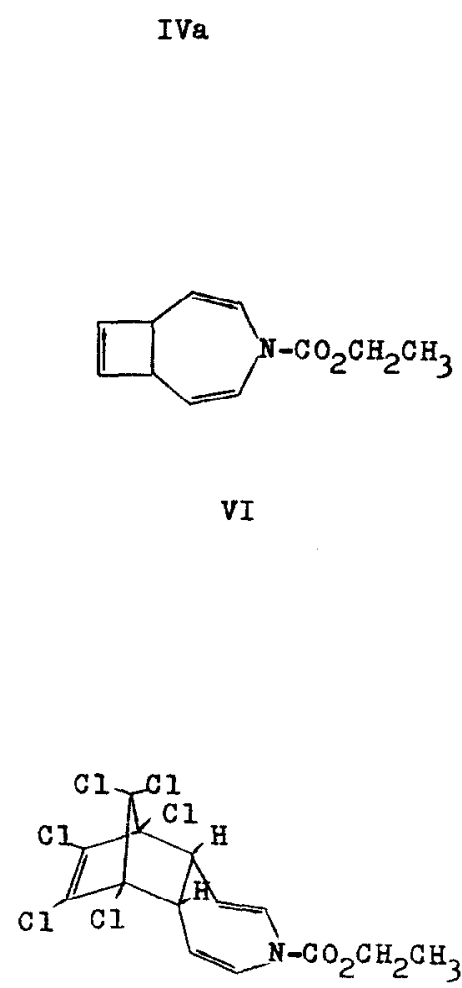

VI

VIII

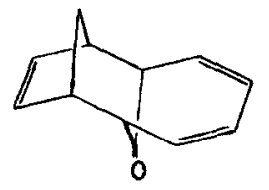

VII 
(2H, multiplet), $\tau 8.78$ ( $5 \mathrm{H}, \mathrm{t}, \mathrm{J}=7 \mathrm{~Hz}$, superimposed on a multiplet). The NMR spectra of the adduct and the tetrahydroadduct when considered together are not in accord with structures IIIa and IVa. The $2 \mathrm{H}$ absorption at $\tau 6.67$ in the NMR spectrum of the adduct III could be logically assigned to the bridgehead protons of IIIa. These protons should appear at higher field in the tetrahydroadduct IV. However, the NMR spectrum of IV shows the lowest field protons at $\tau$ 5.93, which would require a downfield shift. Structures IIIb and IVb are consistent with the NMR spectra. The methine protons in IIIb appear at $\tau 6.67$ and are shifted upfield on hydrogenation, appearing at $\tau 7.21$ in the tetrahydroadduct IVb. The fact that the lowest field absorption integrates for only two protons (disregarding $-0-\mathrm{CH}_{2}-\mathrm{CH}_{3}$ ) is reasonable if the two exo protons a to the nitrogen in IVb are in a different magnetic environment from the two endo protons. This condition is satisfied if the adduct has structure IIIb in the endo configuration.

Confirmation of the spectral assignment was obtained by chemical degradation. The diene II and maleic anhydride were heated at $80^{\circ} \mathrm{C}$ in benzene overnight and a colorless, crystalline anhydride $\mathrm{V}, \mathrm{C}_{11} \mathrm{H}_{8} \mathrm{O}_{5} \mathrm{Cl}_{4}$, mp $96-97^{\circ} \mathrm{C}$, NMR $\tau 6.16(2 \mathrm{H}, \mathrm{s}), \tau 6.39(3 \mathrm{H}, \mathrm{s}), \tau 6.45(3 \mathrm{H}, \mathrm{s})$, IR $\left(1880 \mathrm{~cm}^{-1}\right.$, med; $1795 \mathrm{~cm}^{-1}$, str; $1600 \mathrm{~cm}^{-1}$, med), was isolated from the reaction mixture in 93\% yield. Oxidative cleavage of the adduct III with permanganate/periodate ${ }^{10}$ at room temperature and subsequent sublimation of the product yielded a compound whose $\mathbb{N} M \mathbb{R}$ and IR spectra were identical to those of $V$. Thus, the spectral and chemical evidence estabilsh the structure of the adduct as IIIb, formed by a [4+2] cycloaddition in which the central double bond of $\mathrm{N}$-ethoxycarbonylazepine behaves as a dienophile.

N-ethoxycarbonylazepine and hexachlorocyclopentadiene gave a similar adduct VIII, $\mathrm{C}_{14} \mathrm{H}_{11} \mathrm{NO}_{2} \mathrm{Cl}_{6}, \mathrm{mp} 103.5-104.0^{\circ} \mathrm{C}$, in $40 \%$ yield. The spectra of the adduct, $\operatorname{NMR} \tau 3.01$ (2H, d, J=l1Hz), $\tau 5.07$ (2H, broad d, J=11Hz), $\tau 5.74$ (2H, q, J=7Hz), $\tau 6.43\left(2 \mathrm{H}\right.$, broad s), $\tau 8.68(3 \mathrm{H}, t, J=7 \mathrm{~Hz})$, IR $\left(1740 \mathrm{~cm}^{-1}\right.$, str; $1695 \mathrm{~cm}^{-1}$, str; $1640 \mathrm{~cm}^{-1}$, str; $1605 \mathrm{~cm}^{-1}$, med), show that it has the same type of structure as the ketal adduct III. In particular, the NMR spectra of III and VIII are 
practically superimposable except for the absorption due to the methyl groups of III.

Several attempts to carry out a similar cycloaddition with cyclopentadiene yielded only cyclopentadiene dimer and azepine dimers. Tetracyclone also failed to react.

Acknowledgement. This research was supported by a Frederick Gardner Cottrell Grant from the Research Corporation and by the University of Michigan through a grant from the Office of Research Administration.

\section{References}

1. J. H. Van den Hende and A. S. Kende, Chem. Comm., 1265, 384.

2. L. A. Paquette and J, H. Barrett, J. Am. Chem. Soc, 88, 2590 (1966).

3. A. L. Johnson and H. E. Simmons, J. Am. Chem. Soc., 88, 2591 (1966).

4. K. Hafner and J. Mondt, Angew. Chem., Int. Ed., 2, 839 (1966).

5. W. S. Murphy and J. P. McCarthy, Chem. Comm., 1268, 1155.

6. Leo A. Paquette, Donald E. Kuhla, James H. Barrett, and Louis M. Leichter have reached similar conclusions regarding the reactivity of the 4,5 double bond of $\mathrm{N}$-ethoxycarbonylazepine using different dienes than we have employed. We thank Professor Paquette for informing us of his results prior to publication.

7. All new compounds gave acceptable elemental analyses.

8. S. Masamune and N. T. Castellucci, Angew. Chem., Int. Ed., 3, 582 (1964).

9. R. C. Cookson, B. V. Drake, J. Hudek, and A. Morrison, Chem. Comm., 1966, 15.

10. R. U. Lemieux and E. Von Rudloff, Can. J. Chem., 33, 1701 (1955). 